Supporting Information for

\title{
A diverse family of layered frustrated magnets with tailorable inter-layer interactions
}

Wenjiao Yao, ${ }^{a}$ Lucy Clark, $^{\mathrm{b}}$ Mingjun Xia, ${ }^{\mathrm{c}}$ Teng Li, ${ }^{\text {, }}$, Stephen L. Lee, ${ }^{\mathrm{d}}$ and Philip Lightfoot*a

a. School of Chemistry and EaStChem, University of St Andrews, St Andrews, Fife, KY16 9ST, U.K.

b. Departments of Chemistry and Physics, School of Physical Sciences, University of Liverpool, Liverpool, L69 7ZD, UK.

c. Beijing Center for Crystal Research and Development, Key Laboratory of Functional Crystals and Laser Technology, Technical Institute of Physics and Chemistry, Chinese Academy of Sciences, Beijing 100190, China

d. School of Physics and Astronomy, University of St Andrews, St Andrews, Fife, KY16 9SS, U.K.

*E-mail:pl@st-andews.ac.uk 


\section{Table of contents}

1. Experiments

2. Crystallographic data for compounds $\boldsymbol{I}, \boldsymbol{I I}$ and $\boldsymbol{I I I}$. Table S1

3. Structural representations of compounds $\boldsymbol{I}, \boldsymbol{I I}$ and $\boldsymbol{I I I}$. Figure S1

4. Powder XRD of compound III. Figure S2

5. Fourier-transform infrared (FTIR) patterns for $\boldsymbol{I}, \boldsymbol{I I}$, and $\boldsymbol{I I I}$. Figure S3

6. Thermogravimetric analysis for $\boldsymbol{I}, \boldsymbol{I I}$, and $\boldsymbol{I I I}$. Figure S4

7. Atomic parameters and selected bond information for $\boldsymbol{I}$. Table S2

8. Atomic parameters and selected bond information for $\boldsymbol{I I}$. Table S3

9. Atomic parameters and selected bond information for III. Table S4

10. Polar and nonpolar layers in Type-2 compounds. Figure S5

11. SEM of as-grown $\boldsymbol{I}$. Figure S6

12. Channels in compound $\boldsymbol{I}$. Figure S7

13. References 


\section{Experiments.}

Synthesis: Single crystals of the compounds $\boldsymbol{I}, \boldsymbol{I I}$ and $\boldsymbol{I I I}$ were synthesised by hydrothermal methods using $23 \mathrm{~mL}$ teflonlined autoclaves. Starting materials from commercial companies were applied without further processing. For $\boldsymbol{I}$, $\mathrm{FeC}_{2} \mathrm{O}_{4} \cdot 2 \mathrm{H}_{2} \mathrm{O}$, oxalic acid, $\mathrm{KBF}_{4}$ and $\mathrm{K}_{2} \mathrm{CO}_{3}$ were sealed within the autoclave with $2 \mathrm{~mL}$ distilled water in a molar ratio of 1:3:2:4. Reactions were performed at $160-190{ }^{\circ} \mathrm{C}$ for two days. For $\boldsymbol{I I}, \mathrm{FeC}_{2} \mathrm{O}_{4} \cdot 2 \mathrm{H}_{2} \mathrm{O}$, oxalic acid, sulfuric acid and $\mathrm{Na}_{2} \mathrm{CO}_{3}$ were reacted with $0.5 \mathrm{~mL}$ distilled water in a molar ratio of 10:15:10:2. Reactions were performed at $190{ }^{\circ} \mathrm{C}$ for four days. For III, $\mathrm{FeCl}_{2} \cdot 2 \mathrm{H}_{2} \mathrm{O}$, oxalic acid, and $\mathrm{Li}_{2} \mathrm{CO}_{3}$ were reacted with $5 \mathrm{~mL} 1 \mathrm{M}$ acetic acid in a molar ratio of 1:1:3. The autoclave was kept at $120^{\circ} \mathrm{C}$ for four days. After heating, the autoclaves were cooled down in air. The resulting products were repeatedly washed with distilled water and acetone and dried at $60^{\circ} \mathrm{C}$. Similar procedures were carried out with variants of reactant ratios, water, temperature and time. Unsuccessful reactions typically produced yellow $\mathrm{FeC}_{2} \mathrm{O}_{4} \cdot 2 \mathrm{H}_{2} \mathrm{O}$ crystallites.

Single crystal X-ray diffraction. Transparent crystallites were selected and mounted on nylon loops in inert oil and SXRD data were collected on a Rigaku SCX Mini diffractometer using Mo K $\alpha$ radiation $(\lambda=0.71073 \AA)$ at $173 \mathrm{~K}$. Rigaku CrystalClear 2.0 was employed to index and process the data. Structures were then solved by direct methods and refined using SHELX-2014 ${ }^{1}$ incorporated in the WinGX program. Absorption corrections were performed semi-empirically from equivalent reflections on the basis of multi-scans. Non-H atoms were refined anisotropically.

Characterisation: Fourier-transform infrared spectroscopy was carried out with the objective of determining the coordination of atoms in the sample and to detect any incorporation of $\mathrm{H}_{2} \mathrm{O}$ or $\mathrm{OH}^{-}$. The mid-infrared spectrum was obtained at room temperature using a Perkin Elmer Spectrum GX IR spectrometer. The spectra were collected in the range 400 to $4000 \mathrm{~cm}^{-1}$ with a resolution of $1 \mathrm{~cm}^{-1}$. Powder X-ray diffraction PXRD patterns were recorded on a Stoe STADI/P diffractometer operating in transmission mode with $\mathrm{Fe} \mathrm{K} \alpha_{l}$ radiation $(\lambda=1.936 \AA)$ in the $2 \theta$ range $10^{\circ}-100^{\circ}$. Step scan mode, step size $0.4^{\circ}$, steps time $250 \mathrm{~s}$. Data sets were refined by conventional Rietveld methods using the GSAS package with the EXPGUI interface. ${ }^{2}$ The refinement of the orthorhombic $C m c 2_{1}$ structural model of $\mathrm{KFe}\left(\mathrm{C}_{2} \mathrm{O}_{4}\right) \mathrm{F}$ to room temperature PXRD data yields lattice constants $a=7.7802(1) \AA, b=11.8831(2) \AA$ and $c=10.4216(2) \AA$. The background, scale factor, zero point, lattice parameters and coefficients for the peak shape function were refined to convergence. Thermogravimetric analysis (TGA) were carried out with hand-ground crystals using NETZSCH TG 209 thermal analyser. Samples (about $5 \mathrm{mg}$ ) of ground crystallites were placed in an alumina crucible and heated from room temperature until no obvious weight loss at a rate of $5^{\circ} \mathrm{C} / \mathrm{min}$ in flowing air atmosphere. The residues were examined and analysed by PXRD, all of which showed a typical pattern of $\mathrm{Fe}_{2} \mathrm{O}_{3}$. Small crystallites of $\boldsymbol{I I}$ and $\boldsymbol{I I I}$ were dehydrated at 110 and $190{ }^{\circ} \mathrm{C}$ (assuming the loss of free water molecules) and subjected to SXRD, but no reasonable structure could be determined. SEM images of $\boldsymbol{I}$ were taken on a JEOL JSM-6700F equipped with a field emission gun (FEG) electron source.

\section{Magnetism}

Magnetic susceptibility data were taken on a Quantum Design MPMS SQUID magnetometer in zero-field-cooled (ZFC) field-cooled (FC) cycles over the temperature range $2-300 \mathrm{~K}$ in a $0.01 \mathrm{~T}$ field. The inverse magnetic susceptibilities were analyzed in terms of the Curie-Weiss law, $\chi=C /(T-\theta)$, where $\theta$ and $C$ are the Weiss and Curie constants, respectively. Fitting the inverse susceptibility of $\boldsymbol{I}$ over the temperature range $100-300 \mathrm{~K}$ gave $\theta=-301(2) \mathrm{K}$ and $C=3.757(3) \mathrm{emu} \mathrm{mol}^{-}$ ${ }^{1} \mathrm{~K}$, which corresponds to an effective magnetic moment of $\sim 5.5 \mu_{B}$ per Fe. Between $25-300 \mathrm{~K}$ the magnetic susceptibility can be well modelled in terms of an $S=21$ D Heisenberg spin chain ${ }^{3}$ with an intra-chain coupling constant, $J=8.46(4) \mathrm{K}$. 
2. Crystallographic data for Compounds $\boldsymbol{I}, \boldsymbol{I I}$ and $\boldsymbol{I I I}$

Table S1. Crystallographic data for Compounds I-III

\begin{tabular}{|c|c|c|c|}
\hline & $I$ & II & III \\
\hline Formula & $\mathrm{KFe}\left(\mathrm{C}_{2} \mathrm{O}_{4}\right) \mathrm{F}$ & $\mathrm{Li}_{2} \mathrm{Fe}_{2}\left(\mathrm{C}_{2} \mathrm{O}_{4}\right)_{2} \mathrm{Cl}_{2} \cdot 4 \mathrm{H}_{2} \mathrm{O}$ & $\mathrm{Na}_{2} \mathrm{Fe}\left(\mathrm{C}_{2} \mathrm{O}_{4}\right) \mathrm{SO}_{4} \cdot \mathrm{H}_{2} \mathrm{O}$ \\
\hline Colour & red & dark orange & light orange \\
\hline $\operatorname{Mr}(\mathrm{g} / \mathrm{mol})$ & 201.97 & 444.58 & 303.93 \\
\hline Crystal system & orthorhombic & orthorhombic & hexagonal \\
\hline Space group & $\mathrm{Cmc2}_{1}($ No.36) & $P c a 2_{1}(N o .29)$ & P-62m (No.189) \\
\hline$a(\AA)$ & $7.7385(5)$ & $11.449(2)$ & $10.354(6)$ \\
\hline$b(\AA)$ & $11.8407(8)$ & $11.435(2)$ & \\
\hline$c(\AA)$ & $10.3875(7)$ & $10.144(2)$ & $6.487(4)$ \\
\hline$V\left(\AA^{3}\right)$ & $951.80(11)$ & $1328.0(5)$ & $602.3(9)$ \\
\hline$Z$ & 8 & 4 & 3 \\
\hline $\mathrm{R}$ indices (all data) & $0.0533 / 0.1105$ & $0.0680 / 0.0839$ & $0.0375 / 0.0948$ \\
\hline $\mathrm{R}$ indices $[I>2 \sigma(I)]$ & $0.0335 / 0.0921$ & $0.0395 / 0.0738$ & $0.0334 / 0.0911$ \\
\hline Goodness-of-fit on $\mathrm{F}^{2}$ & 0.769 & 1.051 & 1.153 \\
\hline CCSD No. & 1477442 & 1425817 & 1540906 \\
\hline
\end{tabular}




\section{Structure of compounds $\boldsymbol{I}, \boldsymbol{I I}$ and $\boldsymbol{I I I}$}

(a)

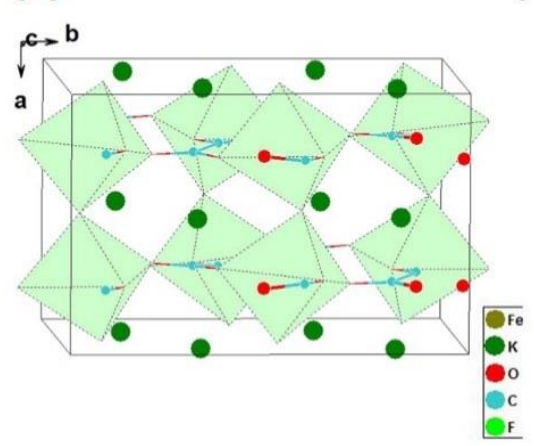

(b)

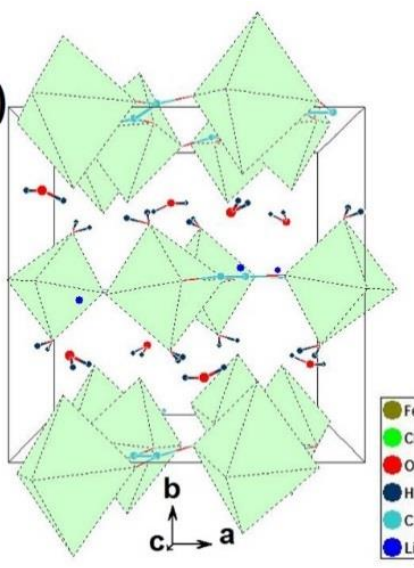

(c)

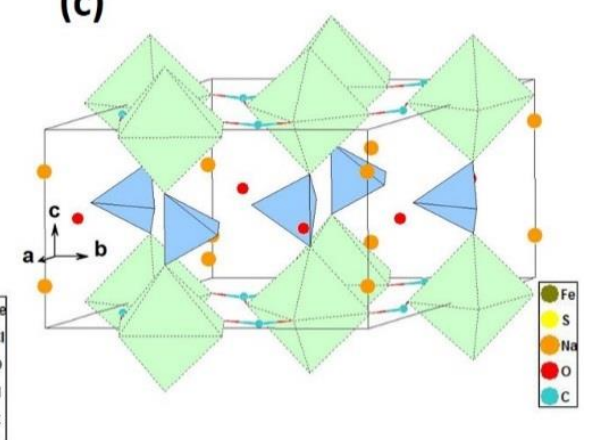

Figure S1. Unit cell of compounds I-III. The green polyhedra indicate Fe-centred octahedra, the blue ones $\mathrm{SO}_{4}$ tetrahedra.

Figure S1 display unit cells for $\boldsymbol{I}$-III. In compound $\boldsymbol{I}$, the Fe is further coordinated to two out-of-plane $\mathrm{F}^{-}$ions, forming a compressed $\left[\mathrm{FeO}_{4} \mathrm{~F}_{2}\right]$ octahedron. The adjacent $\left[\mathrm{FeC}_{2} \mathrm{O}_{4}\right]_{\infty}$ layers are bridged together by sharing $\mathrm{F}^{-}$ions to build a threedimensional framework, with the layers fully overlapped along the $c$-axis. $\mathrm{K}^{+}$ions are located within the channels running in the [100], [010], and [013] directions (discussed below). In II, $\mathrm{Fe}^{2+}$ ions in adjacent layers have different coordination environments, one by $\mathrm{Cl}^{-}$ion to form an elongated $\left[\mathrm{FeO}_{4} \mathrm{Cl}_{2}\right]$ octahedron, the other by $\mathrm{O}$ atoms from water molecules to form an irregular distorted $\left[\mathrm{FeO}_{4}\left(\mathrm{OH}_{2}\right)_{2}\right]$ octahedron. The adjacent layers have no direct connection, resulting in a twodimensional framework. Moreover, to avoid the strong repulsive force between electronegative $\mathrm{Cl}$ and $\mathrm{O}$, the $\left[\mathrm{FeC}_{2} \mathrm{O}_{4}\right]_{\infty}$ layers stack in a staggered way so that $\mathrm{Cl}^{-}$and coordinated $\mathrm{OH}_{2}$ are as far apart as possible. The $\mathrm{Li}^{+}$ions and free water molecules lie between the layers, stabilising the whole structure via electrostatic attraction. In compound $\boldsymbol{I I I}, \mathrm{Fe}^{2+}$ ions are coordinated to two $\mathrm{O}$ atoms from $\mathrm{SO}_{4}{ }^{2-}$ groups at each side of the layer, forming $\left[\mathrm{FeO}_{6}\right]$ octahedra. $\mathrm{The}_{4} \mathrm{SO}_{4}$ groups bridge the adjacent $\left[\mathrm{FeC}_{2} \mathrm{O}_{4}\right]_{\infty}$ layers together in a fully overlapped way, forming the three-dimensional framework. $\mathrm{Na}^{+}$ions and extra free water molecules sit in the channels running along the [001] direction. According to Bond-Valence Sum (BVS) calculations, iron atoms are all in the $2+$ oxidation state in these compounds. The octahedral distortion ${ }^{4}$ is $1.38 \times 10^{-4}$ for $\left[\mathrm{FeO}_{4} \mathrm{~F}_{2}\right]$ in $\boldsymbol{I}, 3.64 \times 10^{-4}$ for $\left[\mathrm{FeO}_{6}\right]$ in $\boldsymbol{I I}, 3.10 \times 10^{-4}$ for $\left[\mathrm{FeO}_{4}\left(\mathrm{OH}_{2}\right)_{2}\right]$ and $6.7 \times 10^{-4}$ for $\left[\mathrm{FeO}_{4} \mathrm{Cl}_{2}\right]$ in $\boldsymbol{I I I}$. 
4. Powder XRD of compound $\boldsymbol{I I I}$

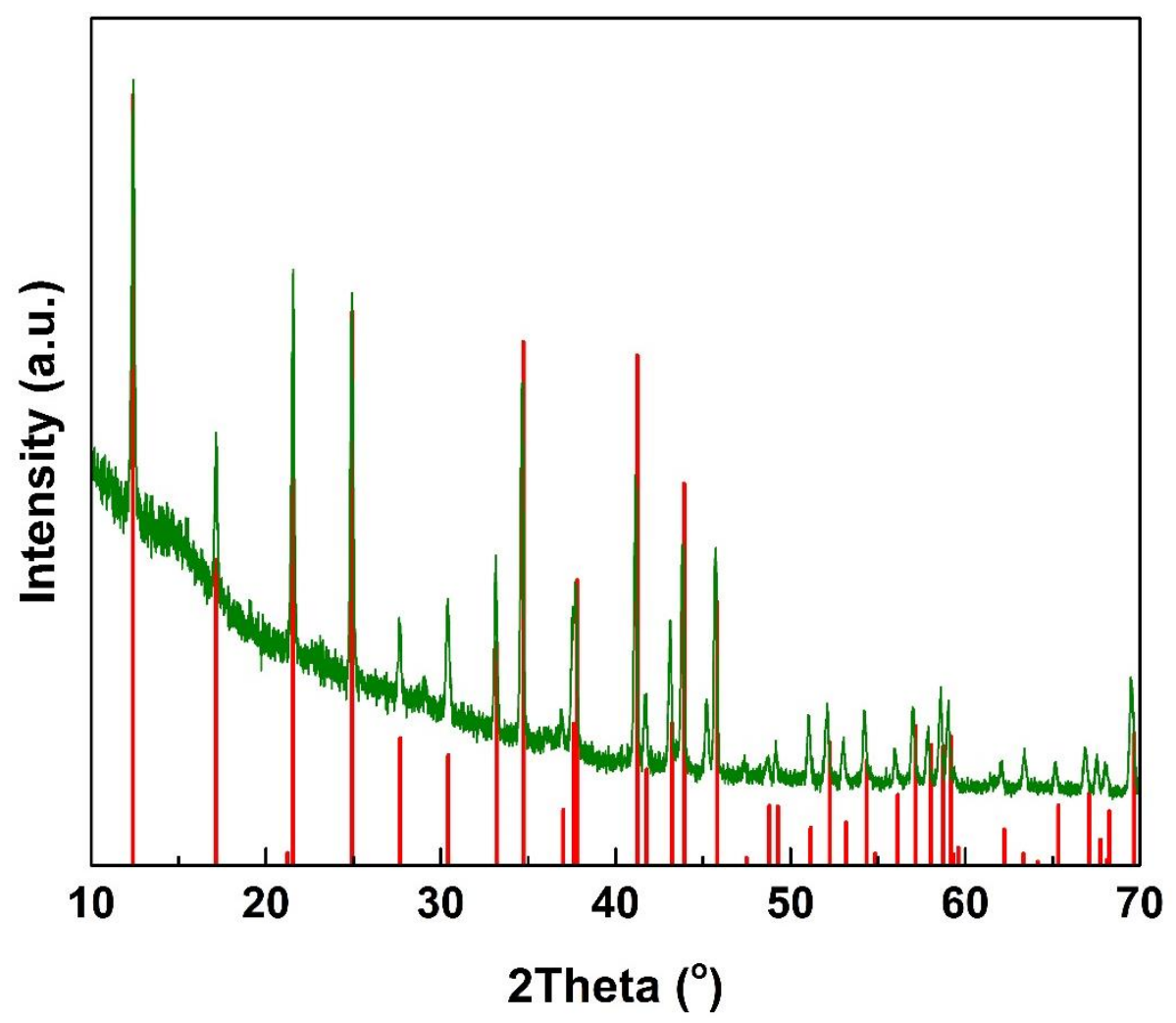

Figure S2. Powder XRD of compound $\boldsymbol{I I I}$. Red lines are theoretical peaks derived from structure determined by single crystal XRD, whereas green line represents experimental powder XRD. 


\section{FTIR patterns for $\boldsymbol{I}, \boldsymbol{I I}$, and $\boldsymbol{I I I}$}
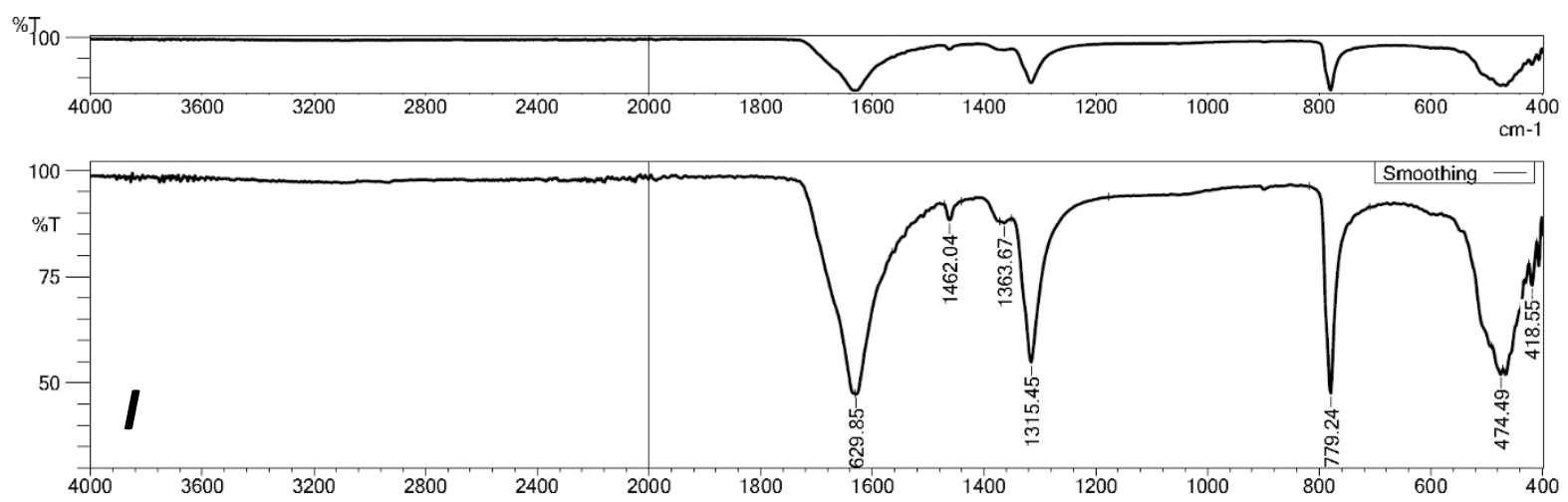

$\% \mathrm{~T}$
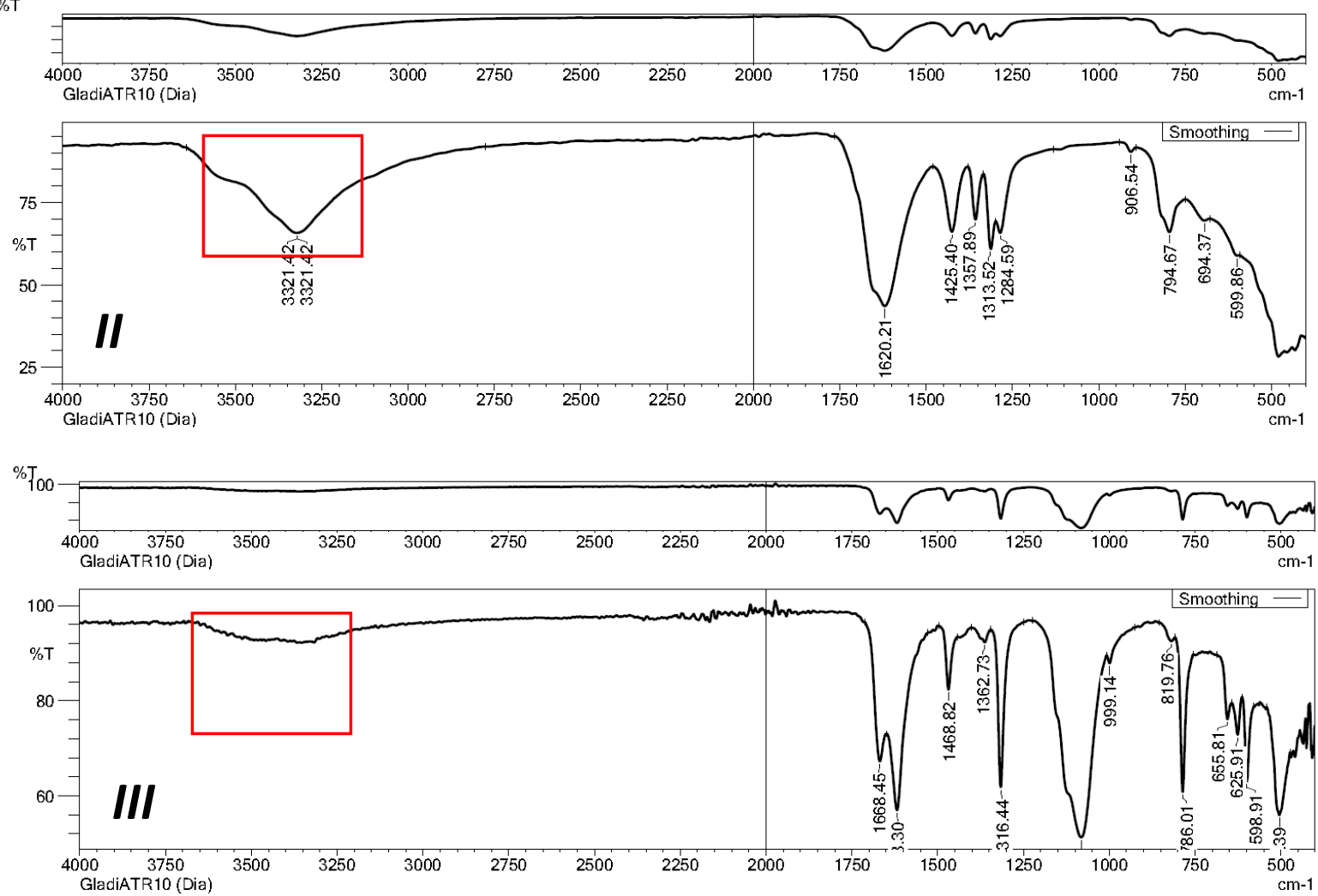

Figure S3. Fourier infrared patterns for $\boldsymbol{I}, \boldsymbol{I I}$, and $\boldsymbol{I I I}$

Wide broad peaks around $3200-3500 \mathrm{~cm}^{-1}$ correspond to $\mathrm{H}-\mathrm{O}$ bonds, as highlighted by red box in the picture. Absorption below $450 \mathrm{~cm}^{-1}$ is not reliable due to facility limitation. Assignments of shorter wavenumber are:

- $<500 \mathrm{~cm}^{-1}$ : Fe-related vibration in $\left[\mathrm{FeX}_{6}\right]$ octahedral;

- 775-800 $\mathrm{cm}^{-1}: \delta(\mathrm{OCO})$ bending modes;

- $900 \mathrm{~cm}^{-1}: \mathrm{v}(\mathrm{C}-\mathrm{C})$ stretching vibration;

Absorptions related to $\mathrm{SO}_{4}$ group are

- $1000-1300 \mathrm{~cm}^{-1}: \mathrm{v}_{\mathrm{ss}}\left(\mathrm{SO}_{4}\right)$ and $\mathrm{v}_{\mathrm{as}}\left(\mathrm{SO}_{4}\right)$;
- $1309 \mathrm{~cm}^{-1}: \mathrm{v}_{\mathrm{s}}(\mathrm{COO})$ involving dangling oxygen;

- $1456 \mathrm{~cm}^{-1}: \mathrm{v}_{\mathrm{s}}(\mathrm{COO})$;

- $\quad 590-630 \mathrm{~cm}^{-1}: \mathrm{vab}_{\mathrm{ab}}\left(\mathrm{SO}_{4}\right)$ 
6. Thermogravimetric analysis for $\boldsymbol{I}, \boldsymbol{I I}$, and $\boldsymbol{I I I}$

TG $/ \%$

DTG /(\%/min)

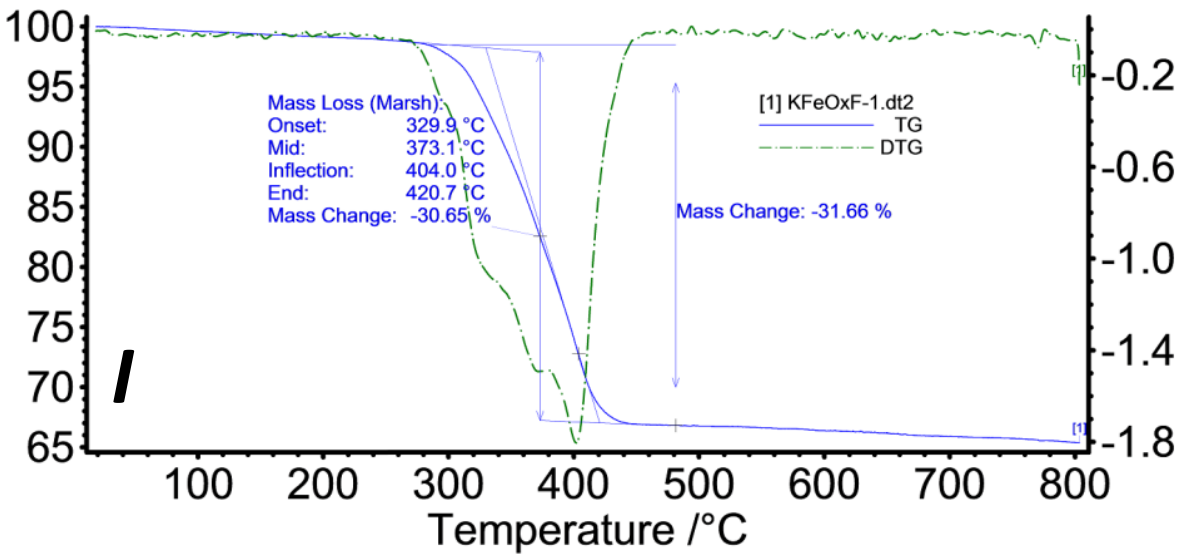

TG $/ \%$

DTG $/(\% / \mathrm{min})$

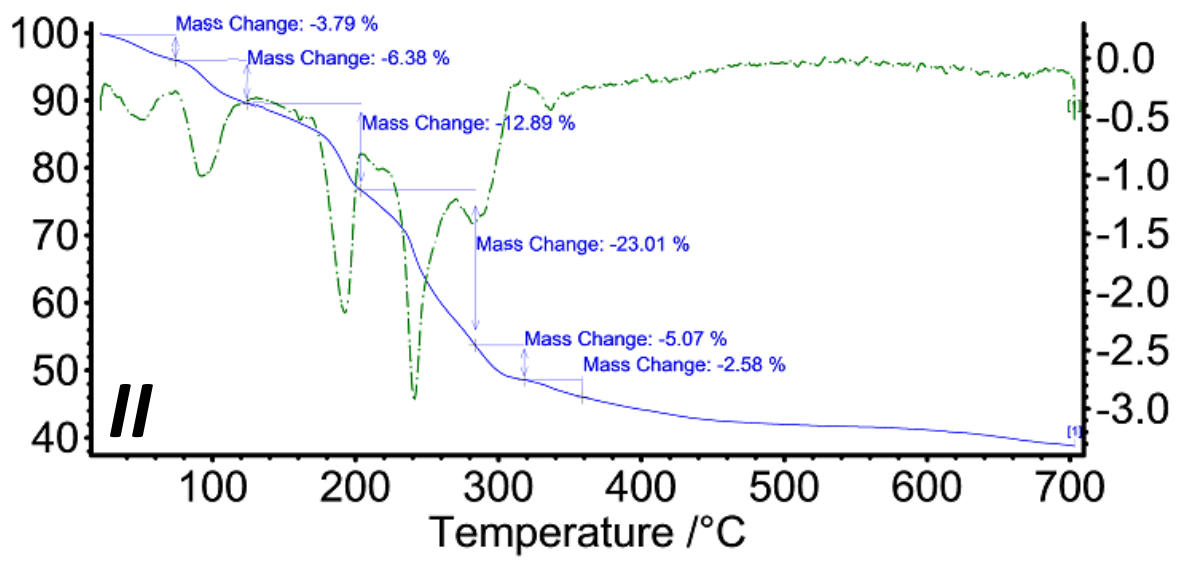

TG $/ \%$

DTG /(\%/min)

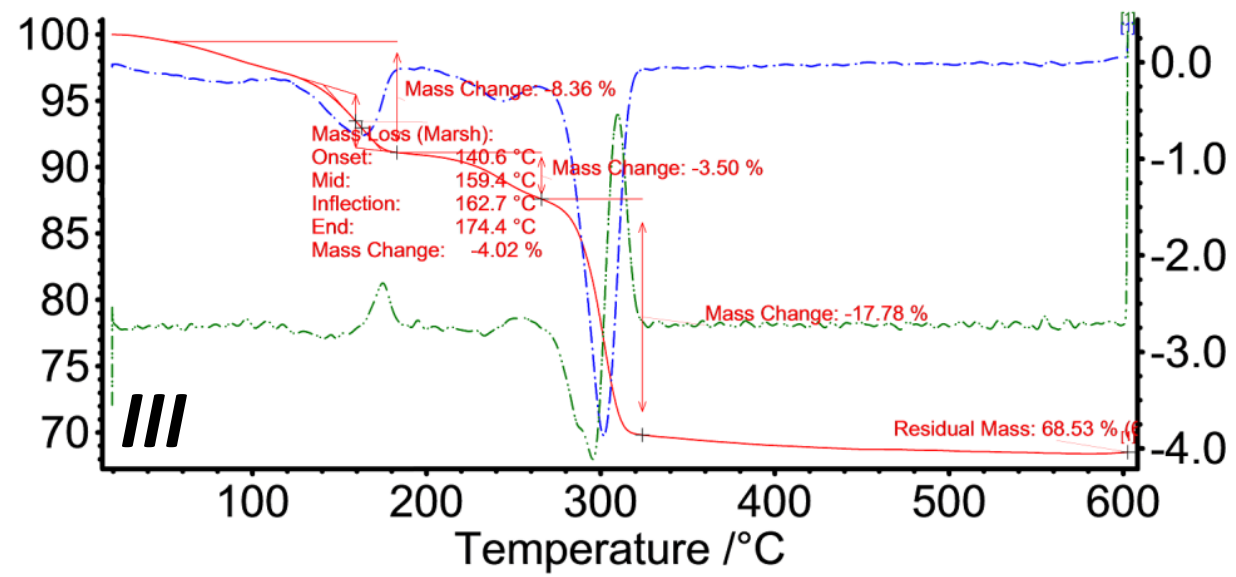

Figure S4. Thermogravimetric analysis for $\boldsymbol{I}, \boldsymbol{I I}$, and $\boldsymbol{I I I}$ 
7. Table S2. Atomic parameters and selected bond information for $\boldsymbol{I}$.

\begin{tabular}{|l|l|l|l|l|l|l|}
\hline Atom & ox. State/BVS & Wyck. & site & x/a & $y / b$ & z/c \\
\hline Fe1 & $+2 /+2.041$ & $8 \mathrm{~b}$ & 1 & $0.24866(16)$ & $0.42682(9)$ & $0.15387(18)$ \\
\hline K1 & $+1 /+1.154$ & $4 a$ & $\mathrm{~m} .$. & $1 / 2$ & $0.3269(2)$ & $0.8727(3)$ \\
\hline K2 & $+1 /+1.133$ & $4 a$ & $\mathrm{~m} .$. & 0 & $0.6600(2)$ & $0.3420(3)$ \\
\hline O1 & -2 & $8 \mathrm{~b}$ & 1 & $0.2453(7)$ & $0.5118(5)$ & $0.4571(7)$ \\
\hline O2 & -2 & $8 \mathrm{~b}$ & 1 & $0.2623(9)$ & $0.3523(6)$ & $0.3420(6)$ \\
\hline O3 & -2 & $8 \mathrm{~b}$ & 1 & $0.2852(8)$ & $0.3905(5)$ & $0.6763(6)$ \\
\hline O4 & -2 & $8 \mathrm{~b}$ & 1 & $0.2891(9)$ & $0.2330(5)$ & $0.5576(7)$ \\
\hline C1 & +3 & $8 \mathrm{~b}$ & 1 & $0.2624(11)$ & $0.4083(7)$ & $0.4450(9)$ \\
\hline C2 & +3 & $8 \mathrm{~b}$ & 1 & $0.2812(11)$ & $0.3369(7)$ & $0.5715(8)$ \\
\hline F1 & -1 & $4 \mathrm{a}$ & $\mathrm{m} .$. & 0 & $0.4570(7)$ & $0.1826(8)$ \\
\hline F2 & -1 & $4 \mathrm{a}$ & $\mathrm{m} .$. & $1 / 2$ & $0.3950(7)$ & $0.1314(8)$ \\
\hline
\end{tabular}

\begin{tabular}{|c|c|c|c|c|c|c|c|}
\hline Atoms 1,2 & d 1,2 [Å] & Atoms 1,2 & d 1,2 [Å] & Atoms 1,2 & d1,2[] & Atoms 1,2 & d 1,2 [Å] \\
\hline $\mathrm{Fe} 1-\mathrm{F} 1$ & $1.980(2)$ & $\mathrm{K} 2-\mathrm{O} 4^{\mathrm{xiv}}$ & $2.903(8)$ & $\mathrm{K} 1-\mathrm{O} 1^{\mathrm{viii}}$ & $2.881(6)$ & $\mathrm{O} 3-\mathrm{C} 2$ & $1.260(11)$ \\
\hline Fe1-F2 & $1.995(2)$ & $\mathrm{K} 2-\mathrm{F} 1$ & $2.919(8)$ & $\mathrm{K} 1-\mathrm{O} 2^{\mathrm{ix}}$ & $2.954(7)$ & O3-Fe1 ${ }^{\text {viii }}$ & $2.194(7)$ \\
\hline $\mathrm{Fe} 1-\mathrm{O} 2$ & $2.146(6)$ & $\mathrm{K} 2-\mathrm{O} 2^{\mathrm{xiii}}$ & $2.928(7)$ & $\mathrm{K} 1-\mathrm{O} 2^{\mathrm{x}}$ & $2.954(7)$ & $\mathrm{O} 3-\mathrm{K} 2^{\mathrm{xvii}}$ & $2.862(7)$ \\
\hline $\mathrm{Fe} 1-04^{\mathrm{i}}$ & $2.160(6)$ & $\mathrm{K} 2-\mathrm{O} 2^{\mathrm{xiv}}$ & $2.928(7)$ & $\mathrm{K} 1-04^{\mathrm{x}}$ & $3.033(8)$ & $\mathrm{O} 4-\mathrm{C} 2$ & $1.24(1)$ \\
\hline $\mathrm{Fe} 1-\mathrm{O} 1^{\mathrm{ii}}$ & $2.170(7)$ & $\mathrm{O} 1-\mathrm{C} 1$ & $1.239(11)$ & $\mathrm{K} 1-04^{\mathrm{ix}}$ & $3.033(8)$ & O4-Fe1 $1^{\text {ix }}$ & $2.160(6)$ \\
\hline $\mathrm{Fe} 1-\mathrm{O}^{\mathrm{ii}}$ & $2.194(7)$ & $01-F e 1^{\text {viii }}$ & $2.170(7)$ & $\mathrm{K} 2-\mathrm{O} 1$ & $2.848(6)$ & $\mathrm{O} 4-\mathrm{K} 2^{\mathrm{xvi}}$ & $2.903(8)$ \\
\hline $\mathrm{K} 1-\mathrm{O}^{\mathrm{v}}$ & $2.737(6)$ & $\mathrm{O} 1-\mathrm{K} 1^{\mathrm{xv}}$ & $2.881(6)$ & $\mathrm{K} 2-\mathrm{O} 1^{\mathrm{xii}}$ & $2.848(6)$ & $\mathrm{O} 4-\mathrm{K} 1^{\mathrm{i}}$ & $3.033(7)$ \\
\hline K1-O3 & $2.737(6)$ & $\mathrm{O} 2-\mathrm{C} 1$ & $1.259(12)$ & $\mathrm{K} 2-\mathrm{O}^{\mathrm{iv}}$ & $2.862(7)$ & $\mathrm{C} 1-\mathrm{C} 2$ & $1.570(2)$ \\
\hline $\mathrm{K} 1-\mathrm{F} 2^{\mathrm{vi}}$ & $2.806(9)$ & $\mathrm{O} 2-\mathrm{K} 2^{\mathrm{xvi}}$ & $2.928(7)$ & $\mathrm{F} 1-\mathrm{Fe} 1^{\mathrm{xii}}$ & $1.980(2)$ & $\mathrm{F} 1-\mathrm{Fe} 1^{\mathrm{xii}}$ & $1.980(2)$ \\
\hline $\mathrm{K} 1-\mathrm{O} 1^{\mathrm{vii}}$ & $2.881(6)$ & $\mathrm{O} 2-\mathrm{K} 1^{\mathrm{i}}$ & $2.954(7)$ & $\mathrm{F} 2-\mathrm{Fe} 1^{\mathrm{v}}$ & $1.995(2)$ & $\mathrm{F} 2-\mathrm{Fe} 1^{\mathrm{v}}$ & $1.995(2)$ \\
\hline Atoms 1,2,3 & $\begin{array}{c}\text { Angle } 1,2,3 \\
{\left[{ }^{\circ}\right]}\end{array}$ & Atoms 1,2,3 & $\begin{array}{c}\text { Angle } 1,2,3 \\
{\left[{ }^{\circ}\right]}\end{array}$ & Atoms 1,2,3 & $\begin{array}{c}\text { Angle } 1,2,3 \\
{\left[{ }^{\circ}\right]}\end{array}$ & Atoms $1,2,3$ & $\begin{array}{c}\text { Angle } 1,2,3 \\
{\left[{ }^{\circ}\right]}\end{array}$ \\
\hline $\mathrm{F} 1-\mathrm{Fe} 1-\mathrm{F} 2$ & $178.0(4)$ & $\begin{array}{c}{ }^{\mathrm{O}} 1^{\mathrm{xii}}-\mathrm{K} 2- \\
\mathrm{O}^{\mathrm{iv}}\end{array}$ & $67.05(17)$ & $\mathrm{F} 2-\mathrm{Fe} 1-\mathrm{O} 2$ & $88.9(3)$ & 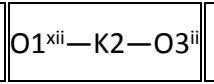 & $129.6(2)$ \\
\hline $\mathrm{F} 1-\mathrm{Fe} 1-\mathrm{O} 2$ & $89.1(3)$ & $\mathrm{O} 1-\mathrm{K} 2-\mathrm{O}^{\mathrm{ii}}$ & $67.05(17)$ & $\mathrm{F} 1-\mathrm{Fe} 1-\mathrm{O} 4^{i}$ & $95.5(3)$ & $\mathrm{O}^{\mathrm{iv}}-\mathrm{K} 2-\mathrm{O} 3^{\mathrm{ii}}$ & $100.9(3)$ \\
\hline $\begin{array}{c}\text { O2-Fe1- } \\
\text { O3 }^{\text {ii }}\end{array}$ & $107.6(2)$ & $\begin{array}{c}\text { O4 }^{\text {xiii }}-\mathrm{K} 2- \\
\text { O4xiv }\end{array}$ & $68.4(3)$ & $\mathrm{O} 4^{\mathrm{i}}-\mathrm{Fe} 1-\mathrm{O}^{\mathrm{ii}}$ & $158.5(3)$ & $\mathrm{O} 1-\mathrm{K} 2-\mathrm{F} 1$ & $74.4(2)$ \\
\hline $\begin{array}{c}\mathrm{O}^{\mathrm{ii}}-\mathrm{Fe} 1- \\
\mathrm{O}^{\mathrm{ii}}\end{array}$ & $76.8(2)$ & $\mathrm{O} 1^{\mathrm{xii}}-\mathrm{K} 2-\mathrm{F} 1$ & $74.4(2)$ & $\begin{array}{c}\mathrm{F} 1-\mathrm{K} 2- \\
\mathrm{O} 2^{\mathrm{xiii}}\end{array}$ & $129.83(17)$ & $\begin{array}{c}\mathrm{O} 1-\mathrm{C} 1- \\
\mathrm{O} 2\end{array}$ & $127.4(9)$ \\
\hline $\mathrm{F} 2-\mathrm{Fe} 1-\mathrm{O} 4$ & $85.0(3)$ & $\mathrm{O} 1-\mathrm{K} 2-\mathrm{O} 4^{\mathrm{xiii}}$ & 59.01(19) & $\mathrm{O} 2-\mathrm{Fe} 1-\mathrm{O} 1^{\mathrm{ii}}$ & $174.8(2)$ & $\mathrm{O} 1-\mathrm{K} 2-\mathrm{O} 4^{\mathrm{xiv}}$ & $103.6(2)$ \\
\hline $\begin{array}{c}\mathrm{O} 2-\mathrm{Fe} 1- \\
\mathrm{O} 4^{\mathrm{i}} \\
\end{array}$ & $93.9(3)$ & $\begin{array}{c}\mathrm{O} 1^{\mathrm{xii}}-\mathrm{K} 2- \\
\mathrm{O} 4^{\mathrm{xiii}}\end{array}$ & $103.6(2)$ & $\mathrm{O} 4^{\mathrm{i}}-\mathrm{Fe} 1-\mathrm{O} 1^{\mathrm{ii}}$ & $81.7(3)$ & $\begin{array}{c}\mathrm{O} 1^{\mathrm{xii}}-\mathrm{K} 2- \\
\mathrm{O} 4^{\mathrm{xiv}}\end{array}$ & 59.01(19) \\
\hline $\begin{array}{c}\text { F1-Fe1- } \\
\text { O1ii }\end{array}$ & $94.0(3)$ & $\begin{array}{c}3^{\text {iv }}-\mathrm{K} 2- \\
\text { O4 }\end{array}$ & $163.7(2)$ & $\mathrm{F} 1-\mathrm{Fe} 1-\mathrm{O}^{\mathrm{ii}}$ & $86.1(3)$ & $\begin{array}{c}3^{\text {iv }}-\mathrm{K} 2- \\
\text { O4 } 4^{\text {iv }}\end{array}$ & $95.32(18)$ \\
\hline $\begin{array}{c}\mathrm{F} 2-\mathrm{Fe} 1- \\
\mathrm{O} 1^{i i}\end{array}$ & $88.0(3)$ & $\begin{array}{c}\mathrm{O}^{\mathrm{ii}}-\mathrm{K} 2- \\
\mathrm{O} 4^{\mathrm{xiii}}\end{array}$ & $95.32(18)$ & $\mathrm{F} 2-\mathrm{Fe} 1-\mathrm{O}^{\mathrm{ii}}$ & $94.2(3)$ & $\begin{array}{c}\mathrm{O}^{\mathrm{ii}}-\mathrm{K} 2- \\
\mathrm{O} 4^{\mathrm{xiv}}\end{array}$ & $163.7(2)$ \\
\hline $\mathrm{O} 2-\mathrm{C} 1-\mathrm{C} 2$ & $115.3(7)$ & $\mathrm{O} 4-\mathrm{C} 2-\mathrm{O} 3$ & $126.8(8)$ & $\mathrm{O} 4-\mathrm{C} 2-\mathrm{C} 1$ & $116.2(8)$ & $\mathrm{O} 3-\mathrm{C} 2-\mathrm{C} 1$ & $117.0(7)$ \\
\hline \multicolumn{8}{|c|}{$\begin{array}{l}\text { (i) } 0.5-x, 0.5-y,-0.5+z ; \text { (ii) } x, 1-y,-0.5+z ; \text { (iii) } x, y,-1+z ; \text { (iv) }-x, 1-y,-0.5+z ; \\
\text { (v) } 1-x, y, z ;(v i) x, y, 1+z ;(\text { vii) } 1-x, 1-y, 0.5+z ;(v i i i) x, 1-y, 0.5+z ; \\
\text { (ix) } 0.5-x, 0.5-y, 0.5+z ;(x) 0.5+x, 0.5-y, 0.5+z ; \text { (xi) } 1-x, y, 1+z ;(x i i)-x, y, z ; \\
\text { (xiii) } 0.5-x, 0.5+y, z ; \text { (xiv) }-0.5+x, 0.5+y, z ;(x v) 1-x, 1-y,-0.5+z ;(x v i) 0.5+x,-0.5+y, z ; \\
\text { (xvii) -x, 1-y, 0.5+z }\end{array}$} \\
\hline
\end{tabular}


8. Table S3. Atomic parameters and selected geometric information for $\boldsymbol{I I}$

\begin{tabular}{|c|c|c|c|c|c|c|c|}
\hline Atom & ox. State/BVS & Wyck. & site & $\mathbf{x} / \mathbf{a}$ & $\mathbf{y} / \mathbf{b}$ & \multicolumn{2}{|l|}{$z / c$} \\
\hline Fe1 & $+2 /+2.056$ & $4 a$ & 1 & $0.82450(7)$ & $0.02337(7)$ & \multicolumn{2}{|l|}{$0.55934(6)$} \\
\hline $\mathrm{Fe} 2$ & $+2 /+2.117$ & $4 a$ & 1 & $0.07146(7)$ & $0.49254(7)$ & \multicolumn{2}{|l|}{$0.41697(7)$} \\
\hline $\mathrm{Cl} 1$ & -1 & $4 a$ & 1 & $0.80804(12)$ & $0.23836(12)$ & \multicolumn{2}{|c|}{$0.59693(14)$} \\
\hline $\mathrm{Cl} 2$ & -1 & $4 a$ & 1 & $0.84496(13)$ & $-0.19162(12)$ & \multicolumn{2}{|c|}{$0.52818(16)$} \\
\hline O1 & -2 & $4 a$ & 1 & $-0.1111(4)$ & $0.5153(3)$ & \multicolumn{2}{|l|}{$0.3961(4)$} \\
\hline $\mathrm{O} 2$ & -2 & $4 a$ & 1 & $0.6395(4)$ & $-0.0005(3)$ & \multicolumn{2}{|l|}{$0.5806(4)$} \\
\hline $\mathrm{O3}$ & -2 & $4 a$ & 1 & $0.7629(4)$ & $0.0320(3)$ & \multicolumn{2}{|l|}{$0.3587(4)$} \\
\hline 04 & -2 & $4 a$ & 1 & $0.2267(3)$ & $0.4720(3)$ & \multicolumn{2}{|l|}{$0.5197(4)$} \\
\hline 05 & -2 & $4 a$ & 1 & $0.1504(4)$ & $0.4691(3)$ & \multicolumn{2}{|l|}{$0.2351(4)$} \\
\hline 06 & -2 & $4 a$ & 1 & $0.8999(4)$ & $0.0037(3)$ & $0.7419(4)$ & \\
\hline 07 & -2 & $4 a$ & 1 & $0.0149(4)$ & $0.5121(3)$ & $0.6189(4)$ & \\
\hline 08 & -2 & $4 a$ & 1 & $0.9821(3)$ & $0.0384(3)$ & $0.4575(4)$ & \\
\hline O9 & -2 & $4 a$ & 1 & $0.1102(4)$ & $0.6768(3)$ & $0.3926(4)$ & \\
\hline 010 & -2 & $4 a$ & 1 & $0.5558(4)$ & $0.2111(3)$ & $0.7213(4)$ & \\
\hline 011 & -2 & $4 a$ & 1 & $0.0492(4)$ & $0.3088(3)$ & $0.4355(4)$ & \\
\hline 012 & -2 & $4 a$ & 1 & $0.1401(4)$ & $0.2784(4)$ & $0.7177(5)$ & \\
\hline $\mathrm{C} 1$ & +3 & $4 a$ & 1 & $1.0856(5)$ & $0.0121(4)$ & $0.4751(6)$ & \\
\hline $\mathrm{C} 2$ & +3 & $4 a$ & 1 & $-0.1659(5)$ & $0.5224(4)$ & $0.5046(6)$ & \\
\hline $\mathrm{C} 3$ & +3 & $4 a$ & 1 & $0.6566(6)$ & $0.0099(4)$ & $0.3483(6)$ & \\
\hline $\mathrm{C} 4$ & +3 & $4 a$ & 1 & $-0.0928(5)$ & $0.5227(4)$ & $0.6308(6)$ & \\
\hline Li1 & $+1 /+1.00$ & $4 a$ & 1 & $0.1688(8)$ & $0.4495(9)$ & $0.7115(10)$ & \\
\hline $\mathrm{Li} 2$ & $+1 /+1.16$ & $4 a$ & \begin{tabular}{l|l}
1 & \\
\end{tabular} & $0.5771(9)$ & $0.0438(9)$ & $0.7642(10)$ & \\
\hline Atoms 1,2 & d 1,2 [Å] & Atoms 1,2 & d 1,2 [Å] & Atoms 1,2 & $d 1,2[]$ & Atoms 1,2 & d 1,2 [Å] \\
\hline Fe1-O6 & $2.055(4)$ & O7-Li1 & $2.121(11)$ & $\mathrm{Fe} 2-\mathrm{O} 1$ & $2.117(4)$ & $\mathrm{C} 3-\mathrm{C} 1^{\mathrm{iii}}$ & $1.542(8)$ \\
\hline $\mathrm{Fe} 1-\mathrm{O} 8$ & $2.087(4)$ & $\mathrm{O} 8-\mathrm{C} 1$ & $1.235(7)$ & $\mathrm{Fe} 2-\mathrm{O} 7$ & $2.160(4)$ & $\mathrm{C} 4-05^{\text {ix }}$ & $1.250(7)$ \\
\hline $\mathrm{Fe} 1-\mathrm{O} 2$ & $2.147(4)$ & $\mathrm{O} 8-\mathrm{Li}^{\mathrm{i}}$ & $2.075(11)$ & $\mathrm{Fe} 2-\mathrm{O} 9$ & $2.167(4)$ & $\mathrm{Li} 1-\mathrm{O} 1^{\mathrm{ix}}$ & $2.026(11)$ \\
\hline $\mathrm{Fe} 1-\mathrm{O} 3$ & $2.156(4)$ & O10-Li2 & $1.977(11)$ & $\mathrm{O} 2-\mathrm{Li} 2$ & $2.058(1)$ & $\mathrm{Li} 1-\mathrm{O}^{\mathrm{x}}$ & $2.096(10)$ \\
\hline $\mathrm{Fe} 1-\mathrm{Cl} 2$ & $2.489(2)$ & O12-Li1 & $1.985(11)$ & $\mathrm{O} 1-\mathrm{C} 2$ & $1.269(7)$ & $\mathrm{Li} 2-\mathrm{O}^{\mathrm{vi}}$ & 2.071(12) \\
\hline $\mathrm{Fe} 1-\mathrm{Cl} 1$ & $2.495(2)$ & $\mathrm{C} 1-\mathrm{O} 2^{\mathrm{vii}}$ & $1.243(8)$ & O1-Li1ii & $2.026(1)$ & Li2-O8 ${ }^{\mathrm{vi}}$ & $2.075(11)$ \\
\hline $\mathrm{O}$-5-C4i & $1.250(7)$ & $\mathrm{C} 1-\mathrm{C}^{\mathrm{vii}}$ & $1.542(8)$ & $\mathrm{O} 2-\mathrm{C} 1^{\mathrm{iii}}$ & $1.243(8)$ & Li2-O6 $6^{\mathrm{iii}}$ & $2.113(11)$ \\
\hline $\mathrm{Fe} 2-\mathrm{O} 5$ & $2.072(4)$ & $\mathrm{C} 2-04^{\text {viii }}$ & $1.241(7)$ & $\mathrm{O} 3-\mathrm{C} 3$ & $1.248(7)$ & $\mathrm{O} 7-\mathrm{C} 4$ & $1.245(7)$ \\
\hline $\mathrm{Fe} 2-\mathrm{O} 4$ & $2.073(4)$ & $\mathrm{C} 2-\mathrm{C} 4$ & $1.530(8)$ & $\mathrm{O} 3-\mathrm{Li}^{\mathrm{i}}$ & $2.071(1)$ & O6-Li2 ${ }^{\mathrm{vii}}$ & $2.113(11)$ \\
\hline Fe2-O11 & $2.125(4)$ & $\mathrm{C} 3-06^{\mathrm{i}}$ & $1.260(8)$ & $\mathrm{O} 4-\mathrm{C}^{\mathrm{iv}}$ & $1.241(7)$ & $\mathrm{O} 6-\mathrm{C} 3^{\mathrm{vi}}$ & $1.260(8)$ \\
\hline Atoms 1,2,3 & $\begin{array}{c}\text { Angle } \\
1,2,3\left[^{\circ}\right]\end{array}$ & Atoms 1,2,3 & $\begin{array}{c}\text { Angle } \\
1,2,3\left[^{\circ}\right]\end{array}$ & Atoms $1,2,3$ & $\begin{array}{c}\text { Angle 1,2,3 } \\
{\left[{ }^{\circ}\right]}\end{array}$ & Atoms 1,2,3 & $\begin{array}{c}\text { Angle } \\
1,2,3\left[^{\circ}\right] \\
\end{array}$ \\
\hline $\mathrm{O} 6-\mathrm{Fe} 1-\mathrm{O} 8$ & $95.29(2)$ & $\mathrm{O} 3-\mathrm{Fe} 1-\mathrm{Cl} 2$ & $87.49(11)$ & $O 5^{i x}-C 4-C 2$ & $114.8(5)$ & $\begin{array}{c}\mathrm{O}^{\mathrm{vii}}-\mathrm{C} 1- \\
\mathrm{C}^{\mathrm{vii}}\end{array}$ & $116.1(5)$ \\
\hline $\mathrm{O} 6-\mathrm{Fe} 1-\mathrm{O} 2$ & $108.03(2) \|$ & $\mathrm{O} 6-\mathrm{Fe} 1-\mathrm{Cl} 1$ & $90.10(11)$ & $\mathrm{O} 8-\mathrm{C} 1-02^{\mathrm{vii}}$ & $128.8(6)$ & O2-Li2-O3 ${ }^{\mathrm{vi}}$ & $95.5(4)$ \\
\hline $\mathrm{O} 8-\mathrm{Fe} 1-\mathrm{O} 2$ & $156.02(2)$ & $\mathrm{O} 8-\mathrm{Fe} 1-\mathrm{Cl} 1$ & $93.42(11)$ & $\mathrm{O} 4^{\mathrm{viii}}-\mathrm{C} 2-\mathrm{C} 4$ & $116.0(5)$ & $\begin{array}{c}4^{\text {viii }}-\mathrm{C} 2- \\
\mathrm{O} 1\end{array}$ & $126.9(6)$ \\
\hline $\mathrm{O} 2-\mathrm{Fe} 1-\mathrm{Cl} 2$ & $88.85(1)$ & $\mathrm{O} 2-\mathrm{Fe} 1-\mathrm{Cl} 1$ & $92.04(10)$ & O5-Fe2-O4 & $93.37(2)$ & $\mathrm{O} 7-\mathrm{C} 4-\mathrm{O} 5^{\text {ix }}$ & $127.7(6)$ \\
\hline (08-Fe1-O3 & $79.13(1)$ & O3-Fe1-Cl1 & $94.26(11)$ & $\mathrm{O5}-\mathrm{Fe} 2-\mathrm{O} 11$ & $90.18(2)$ & O4-Fe2-07 & $78.01(16)$ \\
\hline $\mathrm{O} 2-\mathrm{Fe} 1-\mathrm{O} 3$ & $77.19(2)$ & $\mathrm{Cl} 2-\mathrm{Fe} 1-\mathrm{Cl} 1$ & $178.17(6)$ & $\mathrm{O} 4-\mathrm{Fe} 2-\mathrm{O} 11$ & $86.92(1)$ & \begin{tabular}{|c|}
$\mathrm{O} 11-\mathrm{Fe} 2-$ \\
07 \\
\end{tabular} & $89.00(16)$ \\
\hline $\mathrm{O} 6-\mathrm{Fe} 1-\mathrm{Cl} 2$ & $88.10(1)$ & O6-Fe1-Li2 ${ }^{i}$ & $134.6(2)$ & $\mathrm{O} 5-\mathrm{Fe} 2-\mathrm{O} 1$ & $110.96(2)$ & $\mathrm{O} 1-\mathrm{Fe} 2-07$ & $77.64(17)$ \\
\hline $\mathrm{O} 8-\mathrm{Fe} 1-\mathrm{Cl} 2$ & $86.41(1)$ & $\mathrm{O} 8-\mathrm{Fe} 1-\mathrm{Li}^{\mathrm{i}}$ & $39.5(2)$ & $\mathrm{O} 7-\mathrm{C} 4-\mathrm{C} 2$ & $117.4(5)$ & O5-Fe2-09 & $86.26(16)$ \\
\hline $\mathrm{O} 12-\mathrm{Li} 1-\mathrm{O} 1^{\mathrm{ix}}$ & $96.4(5)$ & $\mathrm{O} 3-\mathrm{Fe} 1-\mathrm{Cl} 2$ & $87.49(11)$ & $011-\mathrm{Fe} 2-\mathrm{O} 1$ & $90.70(1)$ & O4-Fe2-09 & $89.53(15)$ \\
\hline $\mathrm{O} 1-\mathrm{C} 2-\mathrm{C} 4$ & $117.1(5)$ & O3-C3-O6 & $125.7(6)$ & $\mathrm{O} 3-\mathrm{C} 3-\mathrm{C} 1^{\mathrm{iii}}$ & $118.5(5)$ & $\mathrm{O} 6^{\mathrm{i}}-\mathrm{C} 3-\mathrm{C} 1^{\mathrm{iii}}$ & $115.8(5)$ \\
\hline O4-Li1-O7 & $78.9(4)$ & $\mathrm{O} 1^{\mathrm{ix}}-\mathrm{Li} 1-\mathrm{O} 7$ & $94.1(4)$ & O12-Li1-O7 & $102.1(5)$ & $\mathrm{O} 4-\mathrm{Li} 1-\mathrm{O}^{\mathrm{x}}$ & $77.2(4)$ \\
\hline $\mathrm{O} 1^{\mathrm{ix}}-\mathrm{Li} 1-\mathrm{O}^{\mathrm{x}}$ & $101.3(5)$ & $\begin{array}{c}\text { O12-Li1- } \\
\text { O5 }^{\times} \\
\end{array}$ & $105.4(5)$ & $\mathrm{O} 1^{\mathrm{ix}}-\mathrm{Li} 1-\mathrm{O} 4$ & $161.4(6)$ & $\begin{array}{c}\mathrm{O} 12-\mathrm{Li} 1- \\
\mathrm{O} 4\end{array}$ & $101.8(5)$ \\
\hline 舟 & 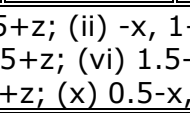 & 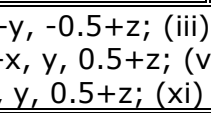 & & (19. & 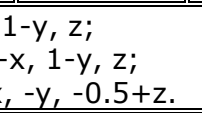 & & \\
\hline
\end{tabular}


9. Table S4. Atomic parameters and selected geometric information for III

\begin{tabular}{|c|c|c|c|c|c|c|c|c|c|c|c|c|}
\hline & Atom & \multicolumn{2}{|c|}{ ox. State/BVS } & Wyck & site & SOF & \multicolumn{2}{|l|}{$x / a$} & \multicolumn{2}{|c|}{$\mathbf{y} / \mathbf{b}$} & \multicolumn{2}{|l|}{$z / c$} \\
\hline & Fe1 & \multicolumn{2}{|c|}{$+2 /+2.089$} & $3 f$ & $\mathrm{~m} 2 \mathrm{~m}$ & & \multicolumn{2}{|c|}{$0.38592(15)$} & \multicolumn{2}{|c|}{1.00000} & \multicolumn{2}{|l|}{1.00000} \\
\hline & S1 & \multicolumn{2}{|c|}{$+6 /+6.135$} & $3 g$ & $\mathrm{~m} 2 \mathrm{~m}$ & & \multicolumn{2}{|c|}{$0.4570(3)$} & \multicolumn{2}{|c|}{1.00000} & $1 / 2$ & \\
\hline & $\mathrm{Na} 1$ & \multicolumn{2}{|c|}{$+1 /+1.301$} & $4 \mathrm{~h}$ & $3 .$. & & \multicolumn{2}{|l|}{$2 / 3$} & \multicolumn{2}{|c|}{1.33330} & $0.7389(6)$ & \\
\hline & O1 & \multicolumn{2}{|c|}{-2} & $6 j$ & m.. & & \multicolumn{2}{|c|}{$0.1508(6)$} & \multicolumn{2}{|c|}{$0.8410(7)$} & 1.00000 & \\
\hline & \multirow{2}{*}{$\begin{array}{l}\mathrm{O} 2 \\
\mathrm{O} 3\end{array}$} & -2 & & $6 j$ & m.. & & $0.1482(7$ & & 0.624 & $0(6)$ & 1.00000 & \\
\hline & & -2 & & $6 \mathrm{k}$ & m.. & & $0.6028(9$ & & 1.132 & $7(8)$ & $1 / 2$ & \\
\hline & 04 & -2 & & $6 i$ &.. $\mathrm{~m}$ & & $0.3696(7$ & & 1.000 & & 0.6817( & \\
\hline & C1 & +3 & & $6 j$ & $\mathrm{~m} .$. & & $0.0884(9$ & & 0.703 & $4(9)$ & 1.00000 & \\
\hline & $\mathrm{Na} 2$ & +1 & & $2 e$ & $3 . \mathrm{m}$ & 0.22 & 1.00000 & & 1.000 & & $0.817(8$ & \\
\hline & $\mathrm{Na} 3$ & \begin{tabular}{l|l}
+1 \\
\end{tabular} & & $3 \mathrm{~g}$ & $\mathrm{~m} 2 \mathrm{~m}$ & 0.52 & $1.197(3)$ & & 1.197 & (3) & $1 / 2$ & \\
\hline Atoms 1, & & d 1,2 [Å] & Atoms & 1,2 & d 1,2 [Å] & & toms 1,2 & & $1,2[]$ & & oms 1,2 & d 1,2 [Å] \\
\hline $\mathrm{Fe} 1-04^{i}$ & & $2.071(6)$ & $\mathrm{Na} 1-\mathrm{N}$ & $1^{\mathrm{vi}}$ & $3.099(8)$ & S1 - & $-04^{v i}$ & 1.48 & $5(6)$ & O3- & $-\mathrm{Na} 3^{\mathrm{xiii}}$ & $3.00(3)$ \\
\hline $\mathrm{Fe} 1-\mathrm{O} 4$ & & $2.071(6)$ & $\mathrm{Na} 1-\mathrm{N}$ & & $3.388(8)$ & $\mathrm{S} 1$ & -04 & 1.48 & $5(6)$ & $\mathrm{C} 1-$ & $\mathrm{C} 1^{\mathrm{xiv}}$ & $1.585(15)$ \\
\hline Fe1-O1 & & $2.152(6)$ & $\mathrm{O} 1-\mathrm{C} 1$ & & $1.235(10)$ & $\mathrm{Na}$ & -03 & 2.40 & $4(6)$ & $\mathrm{Na} 2$ & $-\mathrm{Na} 2^{i}$ & $2.38(10)$ \\
\hline $\mathrm{Fe} 1-\mathrm{O} 1^{\mathrm{ii}}$ & & $2.152(6)$ & $\mathrm{O} 2-\mathrm{C} 1$ & & $1.252(9)$ & $\mathrm{Na}$ & $-03^{\mathrm{vii}}$ & 2.40 & $4(6)$ & $\mathrm{Na} 2$ & $-\mathrm{Na} 2^{\mathrm{vi}}$ & $4.11(10)$ \\
\hline $\mathrm{Fe} 1-\mathrm{O} 2^{\mathrm{iii}}$ & & $2.156(6)$ & $\mathrm{O} 2-\mathrm{Fe}$ & & $2.156(6)$ & $\mathrm{Na}$ & $-03^{\text {viii }}$ & 2.40 & $4(6)$ & $\mathrm{Na3}$ & $-03^{x v}$ & $3.00(3)$ \\
\hline $\mathrm{Fe} 1-\mathrm{O} 2^{\mathrm{iv}}$ & & $2.156(6)$ & $\mathrm{O} 2-\mathrm{Na}$ & & $2.428(5)$ & $\mathrm{Na}$ & $-\mathrm{O} 2^{\mathrm{iii}}$ & 2.42 & $3(5)$ & $\mathrm{Na3}$ & $-03^{x v i}$ & $3.00(3)$ \\
\hline $\mathrm{S} 1-\mathrm{O}^{\mathrm{v}}$ & & $1.447(8)$ & $\mathrm{O} 2-\mathrm{Na}$ & & $2.428(5)$ & $\mathrm{Na}$ & $-02^{i x}$ & 2.42 & $3(5)$ & $\mathrm{Na3}$ & $-\mathrm{Na}^{\mathrm{xiii}}$ & $3.54(5)$ \\
\hline $\mathrm{S} 1-\mathrm{O} 3$ & & $1.447(8)$ & $\mathrm{O} 3-\mathrm{Na}$ & & $2.404(6)$ & $\mathrm{Na}$ & $-O 2^{i i}$ & 2.42 & $3(5)$ & $\mathrm{Na3}$ & $-\mathrm{Na}^{\mathrm{xv}}$ & $3.54(5)$ \\
\hline Atoms 1,2 & & $\begin{array}{c}\text { Angle } 1,2,3 \\
{\left[{ }^{\circ}\right]}\end{array}$ & Atoms & $1,2,3$ & $\begin{array}{c}\text { Angle } 1,2,3 \\
{\left[{ }^{\circ}\right]}\end{array}$ & & oms $1,2,3$ & & $\begin{array}{l}\text { e } 1,2,3 \\
{\left[{ }^{\circ}\right]}\end{array}$ & Atc & ms $1,2,3$ & $\begin{array}{c}\text { Angle } 1,2,3 \\
{\left[{ }^{\circ}\right]}\end{array}$ \\
\hline $\begin{array}{l}\mathrm{O} 4 \mathrm{i}-\mathrm{Fe} 1- \\
\mathrm{O} 4\end{array}$ & & $170.6(4)$ & $03^{v}-S$ & -03 & $110.7(6)$ & $\begin{array}{l}\mathrm{O} 1 \\
\mathrm{O} 2^{\mathrm{i}}\end{array}$ & -Fe1- & 176. & $5(2)$ & $\begin{array}{l}\text { O3 } \\
\text { O3 }^{\text {vii }}\end{array}$ & - $\mathrm{Na} 1-$ & $82.9(2)$ \\
\hline $\begin{array}{l}\mathrm{O} 4 \mathrm{i}-\mathrm{Fe} 1- \\
\mathrm{O} 1\end{array}$ & & $86.49(15)$ & $03^{v}-5$ & $-04^{\mathrm{vi}}$ & $110.3(2)$ & $\mathrm{O}^{\mathrm{i}}$ & -Fe1- & 100. & $5(2)$ & $\mathrm{O}^{\mathrm{O}-}$ & Na1- & $84.52(16)$ \\
\hline $\begin{array}{l}\text { O4-Fe1- } \\
\text { O1 }\end{array}$ & & $86.49(15)$ & $\mathrm{O} 3-\mathrm{S} 1$ & $-04^{v i}$ & $110.3(2)$ & $\begin{array}{l}\mathrm{O} 4^{\mathrm{i}} \\
\mathrm{O} 2^{\mathrm{i}}\end{array}$ & -Fe1- & 93.6 & $9(16)$ & $\begin{array}{l}\mathrm{O}^{\text {vii }} \\
\mathrm{O} 2^{\text {iii }}\end{array}$ & -Na1- & $132.6(2)$ \\
\hline $\begin{array}{l}\mathrm{O} 4{ }^{\mathrm{i}}-\mathrm{Fe} 1- \\
\mathrm{O} 1^{\mathrm{ii}}\end{array}$ & & $86.49(15)$ & $03^{v}-S$ & -04 & $110.3(2)$ & $\mathrm{O} 4$ & -Fe1- & 93.6 & $9(16)$ & $\begin{array}{l}\mathrm{O}^{\text {vii }} \\
\mathrm{O} 2^{\mathrm{ii}}\end{array}$ & -Na1- & $84.52(16)$ \\
\hline $\begin{array}{l}\mathrm{O} 4-\mathrm{Fe} 1- \\
\mathrm{O} 1^{\mathrm{ii}}\end{array}$ & & $86.49(15)$ & $\mathrm{O} 3-\mathrm{S} 1$ & -04 & $110.3(2)$ & $\begin{array}{l}\mathrm{O} 1 \\
\mathrm{O} 2^{\mathrm{i}}\end{array}$ & -Fe1- & 100. & $5(2)$ & $\mathrm{O}^{\mathrm{iii}}$ & -Na1- & 76.69(19) \\
\hline $\begin{array}{l}\text { O1-Fe1- } \\
\text { O1 }{ }^{\mathrm{ii}}\end{array}$ & & $83.0(4)$ & $\mathrm{O} 4^{\mathrm{vi}}-\mathrm{S}$ & -04 & $105.0(5)$ & $\mathrm{O}^{\mathrm{i}}$ & -Fe1- & 176. & $5(2)$ & $\mathrm{O} 2^{\mathrm{ix}}$ & -Na1- & 76.69(19) \\
\hline $\begin{array}{l}\mathrm{O} 4-\mathrm{i}-\mathrm{Fe} 1- \\
\mathrm{O} 2^{\mathrm{iii}}\end{array}$ & & $93.69(16)$ & $\begin{array}{l}\text { O3-Na } \\
\mathrm{O}^{\text {vii }}\end{array}$ & & $82.9(2)$ & $\mathrm{O}^{\mathrm{i}}$ & -Fe1- & 76.1 & & $\begin{array}{l}\mathrm{O3}- \\
\mathrm{Na1}\end{array}$ & $\mathrm{Na1-}$ & $49.87(14)$ \\
\hline $\begin{array}{l}\mathrm{O} 4-\mathrm{Fe} 1- \\
\mathrm{O} 2^{\mathrm{iii}}\end{array}$ & & $93.69(16)$ & $\begin{array}{l}\text { O3-Na } \\
\text { O3 viii }\end{array}$ & & $82.9(2)$ & 01 & $-\mathrm{C} 1-\mathrm{O} 2$ & 127. & 7(7) & $01-$ & $\mathrm{C} 1-\mathrm{C} 1^{\mathrm{xiv}}$ & $116.9(4)$ \\
\hline $\begin{array}{l}\mathrm{O} 1-\mathrm{Fe} 1- \\
\mathrm{O}^{\mathrm{iii}}\end{array}$ & & $176.5(2)$ & $\begin{array}{l}\mathrm{O}^{\text {vii }}-\mathrm{N} \\
\text { O3 }^{\text {viii }}\end{array}$ & $1-$ & $82.9(2)$ & $\mathrm{Na}$ & $-\mathrm{O} 3-$ & & $5.2(8)$ & $\mathrm{O} 3$ & $-\mathrm{C} 2-\mathrm{C} 1$ & $117.0(7)$ \\
\hline $\begin{array}{l}\mathrm{O} 2-\mathrm{C} 1- \\
\mathrm{C} 1^{\text {xiv }}\end{array}$ & & $115.3(4)$ & $\begin{array}{l}\mathrm{O} 3-\mathrm{Na} \\
\mathrm{O} 2^{\mathrm{iii}}\end{array}$ & & & & & & & & & \\
\hline $\begin{array}{l}\text { (i) } x, y, 2-z \text {; } \\
\text { (v) } 1+x-y, 2 \\
\text { (ix) } 1-x, 1-x \\
\text { (xiii) } 2-y, 1\end{array}$ & ; (ii) 1 & $\begin{array}{l}1+x-y, 2-y, 2-z ; \\
1-z ;(\text { ii) } x, y, 1-z \\
2-z ;(x) 1-y, 1+x \\
z ; \text { (xiv) -x, -x+y }\end{array}$ & $\begin{array}{l}\text { (iii) y, 1+x } \\
\text {; (vii) 2-y, } \\
-y, z ;(x i)\end{array}$ & 2-z; (iv) & $\begin{array}{l}-x+y, 1-x, z ; \\
\text { (viii) }-x+y, 2-x \\
-z ;(x i i)-1+y, x\end{array}$ & $\begin{array}{l}x, z \\
x, z\end{array}$ & 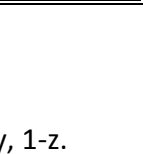 & & & & & \\
\hline
\end{tabular}


10. Polar and nonpolar layers in type-2 compounds

(a)

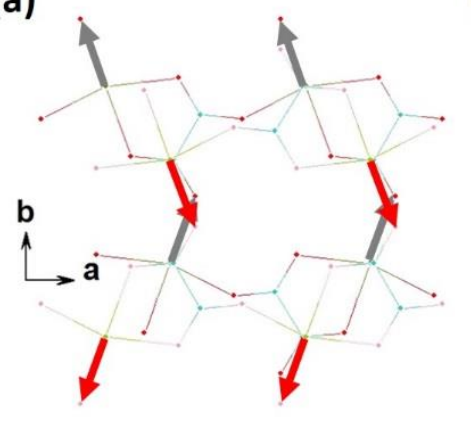

(b)
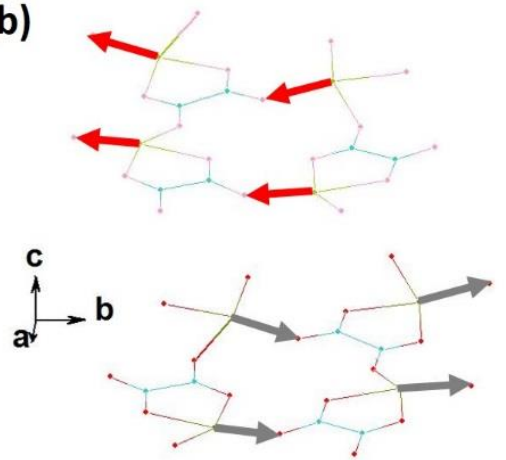

(c)

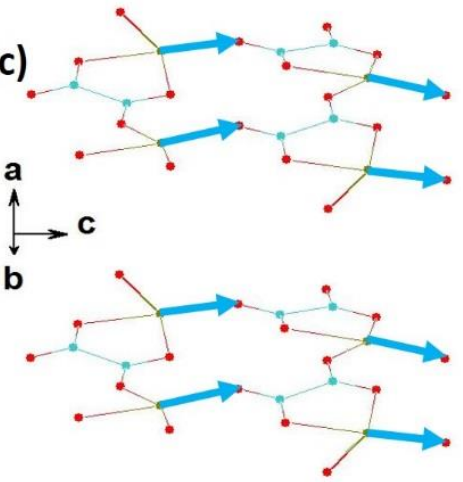

Figure S5. (a-b) Opposite polar directions between neighbouring $\left[\mathrm{Fe}\left(\mathrm{C}_{2} \mathrm{O}_{4}\right)\right]_{\infty}$ layers provide an inversion centre to double layers, as in compound $\boldsymbol{C}$. (c) $\left[\mathrm{Fe}\left(\mathrm{C}_{2} \mathrm{O}_{4}\right)\right]_{\infty}$ layers in compound $\boldsymbol{I}$ polarise in the same direction, resulting in a polar space group. The same for compound $\boldsymbol{I I}$. The arrows indicate the shortest in-plane Fe-O connection. 
11. SEM image of as-grown $\boldsymbol{I}$

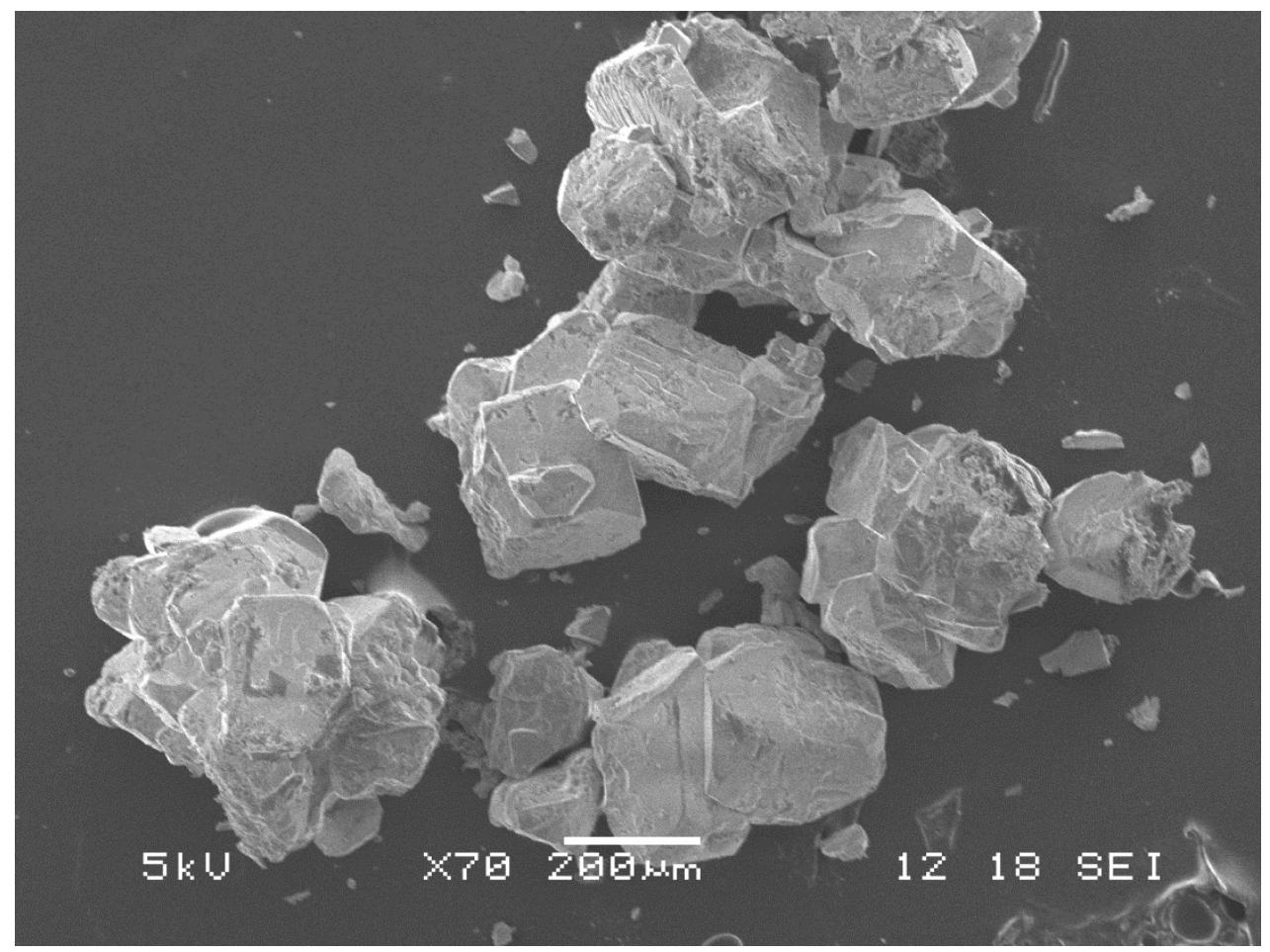

Figure S6. SEM image of as-prepared $I$. 
12. Channels in compound $\boldsymbol{I}$ along [100], [010] and [013] directions
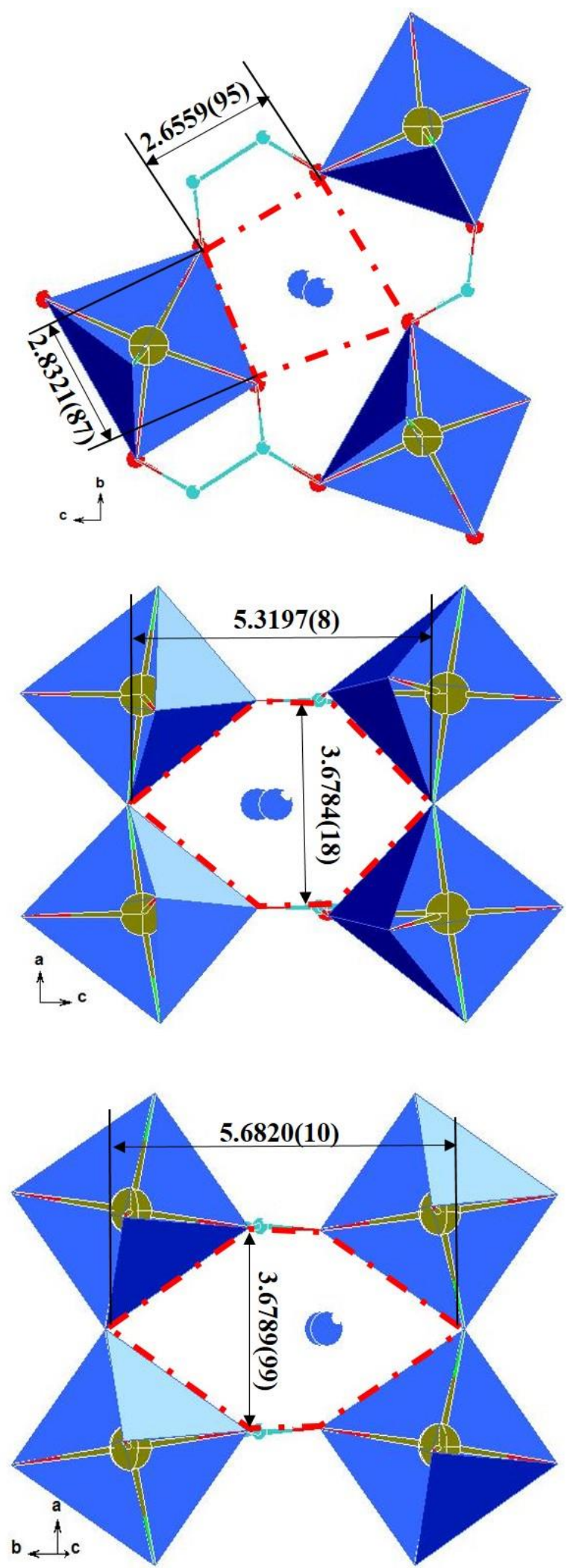

Figure S7. Channels in $\boldsymbol{I}$ along [100], [010] and [013] directions. Dimensions in Å. 
13. References

[1] Sheldrick, G. M. SHELXL-2014, Program for the Refinement of Crystal Structures, University of Göttingen, Göttingen, Germany, 2014.

[2] Toby, B. H. EXPGUI, a graphical user interface for GSAS. J. Appl. Crystallogr. 2001, 34, 210.

[3] Matsushita, Y. and Ueda, Y. Inorg. Chem. 2003, 42, 7830.

[4] Shannon, R. D. Acta Crystallogr. 1976, A32, 751. 